\title{
Experimental study on the effect of hydraulic radius of stack on the performance of thermoacoustic prime mover with open resonator
}

\author{
Yoga N. Adhitama ${ }^{1}$, Danang D. Cahyadi, Ikhsan Setiawan ${ }^{2}$, Agung B. S. Utomo \\ Physics Departement, Faculty of Mathematics and Natural Sciences \\ Universitas Gadjah Mada, Sekip Utara BLS 21 Yogyakarta 55281 Indonesia \\ 1Email : yoga.nur.a@mail.ugm.ac.id, ${ }^{2}$ ikhsan_s@ugm.ac.id
}

Received 21 August 2017, Revised 25 February 2018, Published 31 March 2018

\begin{abstract}
Thermoacoustic prime mover is a device which converts thermal energy into acoustic work. The device mainly consists of a resonator pipe, a stack (a porous medium), and two heat exchangers. Four stacks were made of a pile of stainless wire-mesh screens with various mesh numbers of \#12, $\# 14$, \#16, and \#18, providing the effective hydraulic radius of $0.32 \mathrm{~mm}, 0.28$ $\mathrm{mm}, 0.25 \mathrm{~mm}, 0.23 \mathrm{~mm}$, respectively. Each stack has $4 \mathrm{~cm}$ length. By using the wire-mesh screens, it is easier to vary the hydraulic radius of stack than any other material. The stack is sandwiched between a hot heat exchanger and an ambient heat exchanger. The resonator is made of a stainless-steel pipe with one end open and the other closed. The stack and heat exchangers are placed inside a resonator pipe near the closed end. The thermal energy were provided by using an electric heater which is installed at the hot heat exchanger. Ambient water was flowing through the ambient heat exchanger. Sound is produced by the stack when the temperature difference between the both ends of stack reachs an onset point. This experiments were performed by recording the temperatures at both ends of stack and the pressure amplitudes of the sound at several points along the resonator for various hydraulic radius of stack. It is found that the effective hydraulic radius of $0.28 \mathrm{~mm}$ gives the lowest onset temperature, the shortest time to reach onset condition, and the highest pressure amplitude which are $314^{\circ} \mathrm{C}, 299 \mathrm{~s}$, and $2.89 \mathrm{kPa}$, respectively. The frequency of sound is not altered by the change of hydraulic radius of stack.
\end{abstract}

Keywords: Thermoacoustic prime mover, hydraulic radius, onset temperature difference, onset time, pressure amplitude, sound frequency.

\section{INTRODUCTION}

Electricity that consumed worldwide is mostly obtained from coal combustion process. This process emits a variety of pollutant gases, one of them is carbon dioxide gas $\left(\mathrm{CO}_{2}\right)$. The emission of $\mathrm{CO}_{2}$ and other pollutant gases to the atmosphere triggers an increase in the average temperature of the earth and contributes to climate change around the world. The impact of burning coal needs to be reduced by developing 
alternative energy utilization technologies. One of the 'green' technologies is using thermoacoustic technique.

The first thermoacoustic phenomenon was discovered more than 200 years ago by Byron Higgins in 1777 known as the singing flame. Nevertheless, the term thermoacoustics was first used by Nikolaus Rott in 1980 to describe the study of acoustic effects caused by changes in entropy and heat conduction on a medium (Rott, 1980).

Thermoacoustic devices are divided into two types: thermoacoustic prime mover, which converts thermal energy into acoustic energy, and thermoacoustic refrigerator, which converts acoustic energy into thermal energy. Thermoacoustic prime mover can be used as an electric generator by combining it with linear alternator in the form of piezoelectric elements or magnetic induction (Agarwal et al., 2016). The advantages of thermoacoustic prime movers are environmentally friendly, it does not produce any waste, simple configuration and high durability with relatively low cost of manufacture and maintenance (Setiawan et al., 2015). The thermal energy used can be obtained from various sources such as solar heat (Chen \& Garret, 1998), waste heat from vehicle exhaust gas (Gardner \& Howard, 2009), and cooking stoves (Normah et al., 2013). The working gas used is an environmentally friendly gas, so it is not dangerous in case of leakage. Among those many benefits, the thermoacoustic prime mover has the disadvantages that is low in efficiency, therefore most of the thermoacoustic studies discuss about efforts to optimize the performance of thermoacoustic prime mover devices.

In 2011, Hao et al. conducted an experiment to determine the effect of working fluid on the performance of its twin thermoacoustic prime mover using nitrogen, helium, argon, and mixtures of helium-argon gas as a working fluid. Another example of research on thermoacoustic prime mover was conducted by Hariharan et al. (2015). They examined the effect of geometrical parameters such as the length of the stack and resonator, and also the thickness of the stack on the performance of the thermoacoustic prime mover. Afterwards, they compared the results obtained with the simulation results using DeltaEC (Design Environment for Low Amplitude Thermoacoustic Energy Conversion) software.

This paper describes the effect of stack effective hydraulic radius on the performance of a standing wave thermoacoustic prime mover with open-end resonator. The performance parameters are the onset temperature difference, time needed to reach the onset condition, sound frequency, and amplitude of sound pressure generated.

\section{THEORY}

Thermoacoustic prime mover basically consist of resonators, stacks, and two heat exchangers. Stack is a porous medium in which thermoacoustic effects occur, flanked by hot-heat exchanger (HHX) and cold-heat exchanger (CHX) placed inside the resonator pipe. Prime movers absorb heat $Q_{H}$ from the heat source and then convert some of it into work $W$ in the form of sound waves and release a certain amount of heat $Q_{C}$, as shown in Fig. 1 . 


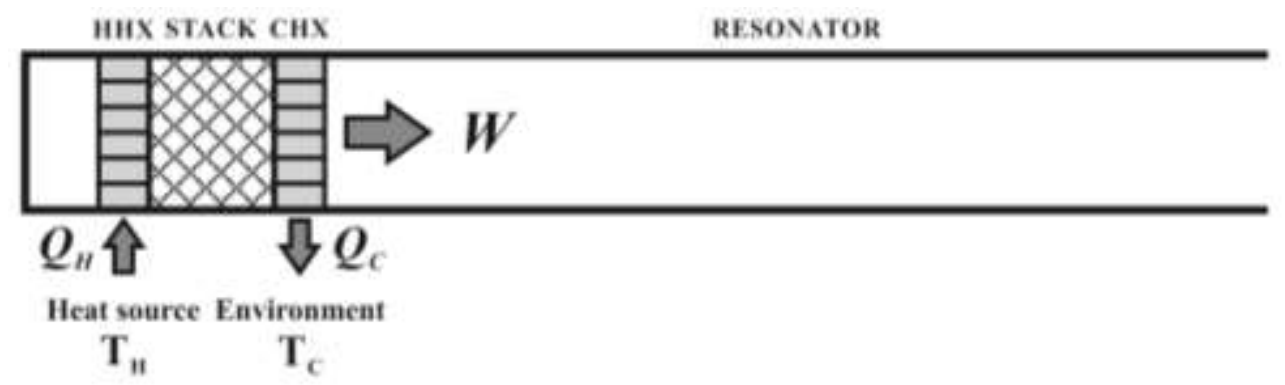

Figure 1. The schematic diagram of the standing wave thermoacoustic prime mover with open-end straight resonator

The net output power of the three main components of the thermoacoustic prime mover can be expressed as (Atchley, 1994)

$$
\dot{W}=\dot{W}_{R E S}+\dot{W}_{S T K}+\dot{W}_{H E} .
$$

The output power on the resonator $\left(\dot{W}_{R E S}\right)$ and heat exchanger $\left(\dot{W}_{H E}\right)$ are dissipations that are always negative, while the output power on the stack $\left(\dot{W}_{S T K}\right)$ can be either positive or negative depending on the temperature gradient across the stack. At first, $\dot{W}_{S T K}$ is negative, but as the temperature difference between the two ends of the stack increases, this value also increases until the stack's output power becomes zero $\left(\dot{W}_{S T K}=0\right)$. At this point, the temperature difference between two ends of the stack is called critical temperature difference $\left(\Delta T_{\text {crit }}\right)$. After the critical condition is reached, the gas package starts to oscillate so that the stack's output power becomes positive, but sound waves cannot be generated because the net output power is still negative ( $\dot{W}<$ $0)$. When the sum of the output power in all three main components becomes positive $(\dot{W}>0)$, the sound wave begins to be generated. This state is called the onset condition, and the temperature difference between the two ends of the stack is called the onset temperature difference $\left(\Delta T_{\text {onset }}\right)$.

The process of generating sound waves on the stack components follows the processes in the Brayton cycle (Jin dkk., 2015), which consists of two isobaric processes and two adiabatic processes, which are schematically illustrated by Fig. 2.(a) and (b).

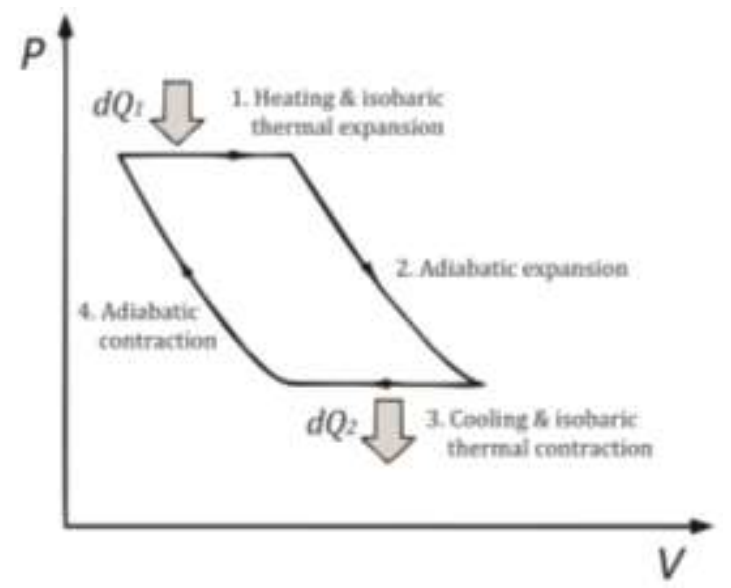

(a) 


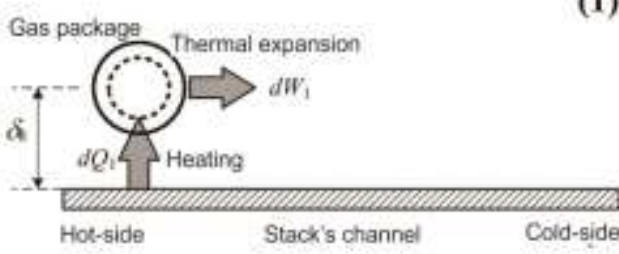

(3)

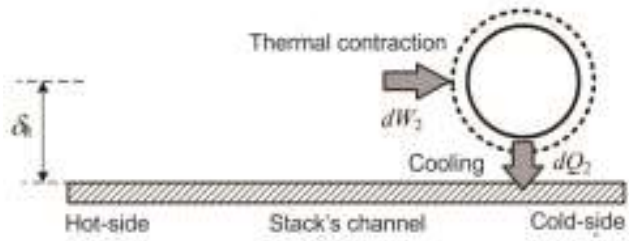

(2)

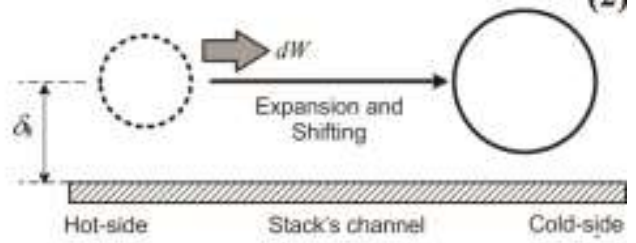

(4)

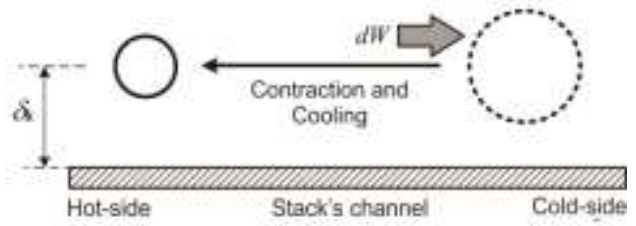

(b)

Figure 2.(a) The four steps taken by the gas package in one cycle. (b) $P-V$ diagram of the Brayton cycle (Setiawan dkk., 2015)

The gas inside the pores of the stack is considered as a gas package. The gas package on the left side (hot section) is heated by absorbing a certain amount of heat $d Q_{1}$ and undergoing thermal expansion, in this case, certain amount of work $d W_{1}$ is done by the gas package. Then due to the expansion process, the gas package does some work $d W$ and spreads to the right side. This makes the pressure on the left side decreased. After reaching the right side, the gas package releases some heat $d Q_{2}$ due to higher temperature than the stack channel, also experiences cooling and thermal contraction. At this rate, some work $d W_{2}$ is done for the gas package. Next, the gas package returns to the left side to fill the low pressure area and undergoes adiabatic compression. At this point, the work $d W$ is carried out on the gas package. Afterwards, the gas package is heated again and the cycle re-started (Setiawan dkk., 2015). These steps continue to repeat until the temperature difference between two ends of the stack reaches $\Delta T_{\text {onset }}$ and the sound waves succesfully generated.

In selecting material for the stack should consider its two geometrical parameters, that is porosity $(\phi)$ and hydraulic radius $\left(r_{h}\right)$. Porosity is defined as the ratio of the open volume (cavity) to the stack overall volume (Abduljalil dkk., 2011).

$$
\phi=\frac{V_{\text {gas }}}{V_{\text {stack }}}=1-\frac{V_{\text {solid }}}{V_{\text {stack }}},
$$

where $V_{\text {gas }}$ and $V_{\text {solid }}$ is part of the stack volume occupied by the gas and solid, respectively. Hydraulic radius represents the ratio of the open volume to the total area of solid material which is in contact with the working gas that can be written as (Abduljalil dkk., 2011)

$$
r_{h}=\frac{V_{\text {gas }}}{A_{\text {contact }}} .
$$

One of the materials that can be used for the stack is a wire mesh. The use of wire mesh as the stack material since it is easily obtained and its geometrical parameters are 
easily varied. Porosity and hydraulic radius of the stack that is made of wire mesh can be estimated using equations (Abduljalil dkk., 2011):

$$
\begin{gathered}
\phi=1-\frac{\pi n d_{\text {wire }}}{4}, \\
r_{h}=d_{\text {wire }} \frac{\phi}{4(1-\phi)},
\end{gathered}
$$

where $n$ is the number of pores per unit length (called mesh number) provided by the manufacturer, while $d_{\text {wire }}$ is diameter of the wire forming the wire mesh. The more precise value of hydraulic radius on stacks made of wire mesh can be calculated using array modelling of circular tubes. Radius of its circular area can be assumed to be the effective hydraulic radius of the stack $\left(r_{e f f}\right)$, can be expressed as (Ueda, 2009)

$$
r_{\text {eff }}=\sqrt{\frac{r_{h} d_{\text {wire }}}{2}} .
$$

Besides of these two geometrical parameters, based on the perpendicular direction of the gas oscillation, there are two other parameters which contribute to the performance of the prime mover, both parameters are thermal penetration depth $\left(\delta_{k}\right)$ and viscous penetration depth $\left(\delta_{v}\right)$. Thermal penetration depth is a measure of how far the heat can diffuse through medium, that can be estimated as

$$
\delta_{k}=\sqrt{\frac{2 k}{\omega \rho_{m} c_{P}}}=\sqrt{\frac{2 \kappa}{\omega}},
$$

where $k$ and $\rho_{m}$ is thermal conductivity and the average density of the gas, $c_{P}$ is the isobaric specific heat, and $\kappa$ is the thermal diffusivity of the gas (Saechan, 2014). Viscous penetration depth can be explained as the thickness of the working fluid layer around the stack whose movement is influenced by the viscous force (Murti, 2015). Viscous penetration depth can be calculated as

$$
\delta_{v}=\sqrt{\frac{2 \mu}{\omega \rho_{m}}}=\sqrt{\frac{2 v}{\omega}},
$$

where $\mu$ and $\nu$ are respectively the dynamic and kinetic viscosities of the working fluid (Saechan, 2014). The material selection of the stack should also consider the Lautrec number $\left(N_{L}\right)$, which is ratio of the effective hydraulic radius to the thermal penetration depth on the stack $\left(N_{L}=r_{e f f} / \delta_{k}\right)$.

\section{EXPERIMENTAL SETUP AND METHOD}

This study uses a resonator made of stainless straight pipe with a length of $155 \mathrm{~cm}$ and $6,8 \mathrm{~cm}$ in diameter, with one end opened and the other end closed. The resonance frequency formed on this resonator pipe is formulated as

$$
f_{m}=(2 m-1) \frac{v_{\text {sound }}}{4 L},
$$

where $L$ is the resonator pipe length, $m$ is the harmonic order $(m=1,2,3, \ldots$ etc $)$, and $v_{\text {sound }}$ represents the speed of sound on free air medium of $347,2 \mathrm{~m} / \mathrm{s}$. 
Heat exchangers are used to transfer the heat from and to the stack, and it is made of copper for optimal heat transfer process. Both heat exchangers have pores parallel to the resonator axis that allow the gas to oscillate through the pores. Thermal energy source used in this research is electric heater with input power of 350 watt wrapped around the hot-heat exchanger. A water circulation system is assembled on the outer wall of the resonator pipe which is in contact to the cold-heat exchanger to keep the temperature stable. Inside the resonator pipe, there are 5 pieces of pressure transducer to measure the pressure amplitude of the sound waves along the resonator, also there are $3 \mathrm{~K}$-type thermocouples to measure the temperature at both ends of the stack $\left(T_{H}\right.$ and $\left.T_{C}\right)$ and the closed-end of the resonator $\left(T_{C E R}\right)$. Location of each pressure transducer and thermocouple along the resonator is shown in Fig. 3.

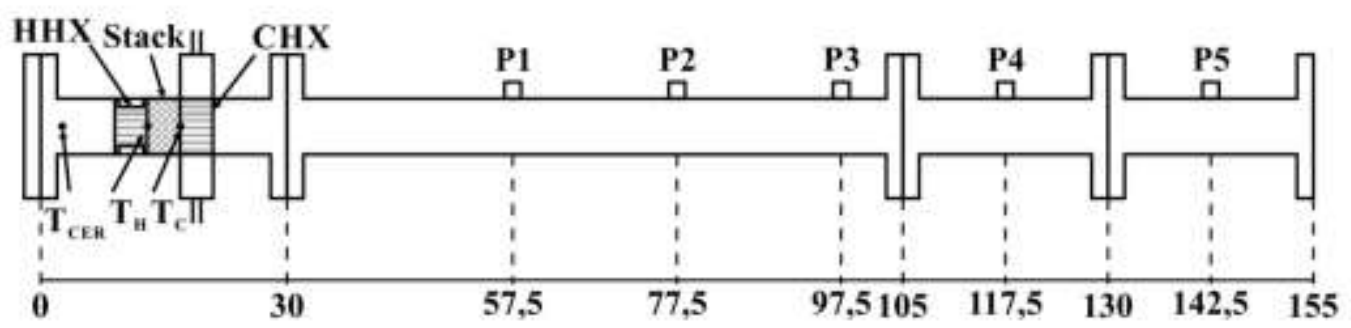

Figure 3. Locations of the pressure transducers and K-type thermocouples

The stack is made from a stacked stainless steel wire mesh with a diameter of $6,8 \mathrm{~cm}$ and $4 \mathrm{~cm}$ in thick. The number of pores of the wire mesh per inch are expressed by the mesh number. Effective hydraulic radius values of the stack can be varied by changing the mesh number used. In this study, the mesh numbers used are \#12, \#14, \#16, and \#18 (Fig. 4). The value of porosity, hydraulic radius, and effective hydraulic radius of stack for each of the mesh number used are summarized in Table 1.
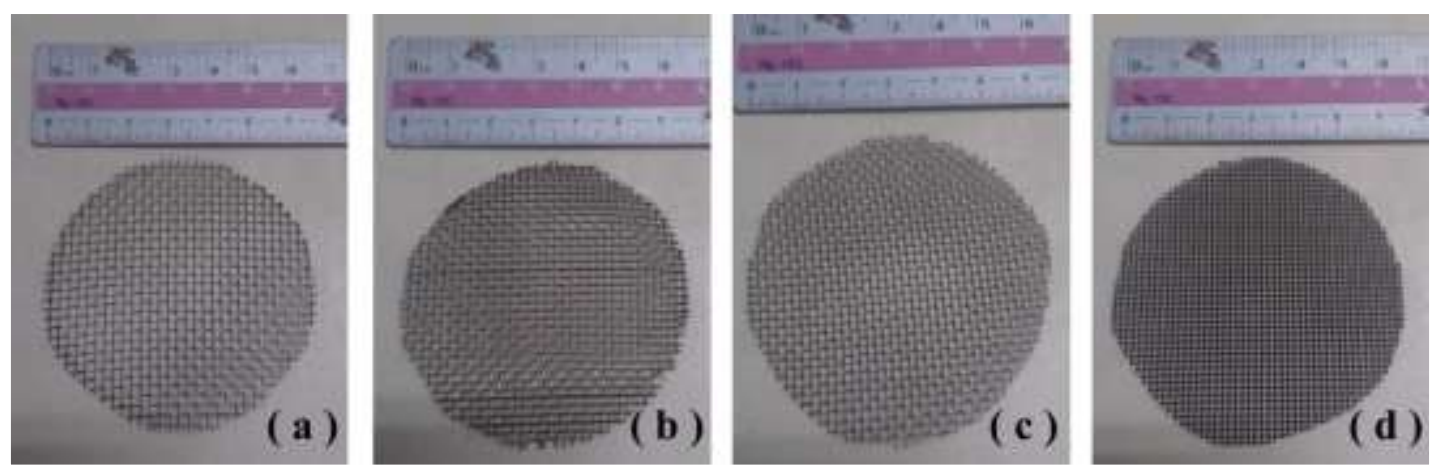

Figure 4. The mesh numbers used for the stack; (a) \#12, (b) \#14, (c) \#16, (d) \#18 
Table 1. The dimension of the wire mesh used

\begin{tabular}{ccccc}
\hline $\begin{array}{c}\text { Mesh } \\
\text { number }\end{array}$ & $d_{\text {wire }}(\mathbf{m m})$ & Porosity (\%) & $r_{h}(\mathbf{m m})$ & $r_{\text {eff }}(\mathbf{m m})$ \\
\hline $\mathbf{1 2}$ & 0,36 & 86,6 & 0,58 & 0,32 \\
$\mathbf{1 4}$ & 0,32 & 86,1 & 0,50 & 0,28 \\
$\mathbf{1 6}$ & 0,29 & 85,6 & 0,43 & 0,25 \\
$\mathbf{1 8}$ & 0,27 & 84,9 & 0,38 & 0,23 \\
\hline
\end{tabular}

Temperatures at both ends of the stack and the closed-end of the resonator begin to be recorded since the electric heater is turned on until the experiment is completed, while the recording of the pressure oscillation data begins shortly after sound waves can be generated. The electric heater is turned off when the temperature on the hot-side $\left(T_{H}\right)$ and the cold-side of the stack $\left(T_{C}\right)$ no longer increased, and the pressure oscillation data recording process is also stopped. Furthermore, the temperature data obtained from the thermocouple was analyzed to find the onset temperature difference. The onset condition is determined when there is a significant increase in temperature on the coldside of the stack. The onset temperature difference is formulated as

$$
\Delta T_{\text {onset }}=T_{H}-T_{C} \text {. }
$$

The pressure oscillation data as a function of time obtained from the pressure transducer is converted into frequency domain (as a frequency spectrum) by Fast Fourier Transform (FFT) method using SciLab software. Based on the resulting frequency spectrum, the information about frequency and amplitude of the sound waves generated can be acquired.

\section{RESULTS AND DISCUSSION}

\subsection{Onset Temperature Difference and Onset Time}

Temperature on the hot-side of the stack increases significantly shortly after the electric heater is turned on. Right at that moment, the heat on the hot-side propagates by convection toward the closed-end of resonator and is reflected off to the cold-side of the stack. This convective propagation causes the temperature at the hot-side of stack and the closed-end of resonator to rise rapidly, while only a few heat reach the cold-side so that the temperature is relatively unchanged. After a while, most of the heat has reached the cold-side of stack and caused a significant rise in temperature along with the generation of sound waves, this point is defined as onset condition. Temperature on the cold-side of stack continues to increase due to convection and conduction, so that the temperature rise between the two stack sides decreases to a relatively constant value. It is in this condition that the thermal equilibrium (also called steady state) is reached on every parts of the thermoacoustic prime mover. 


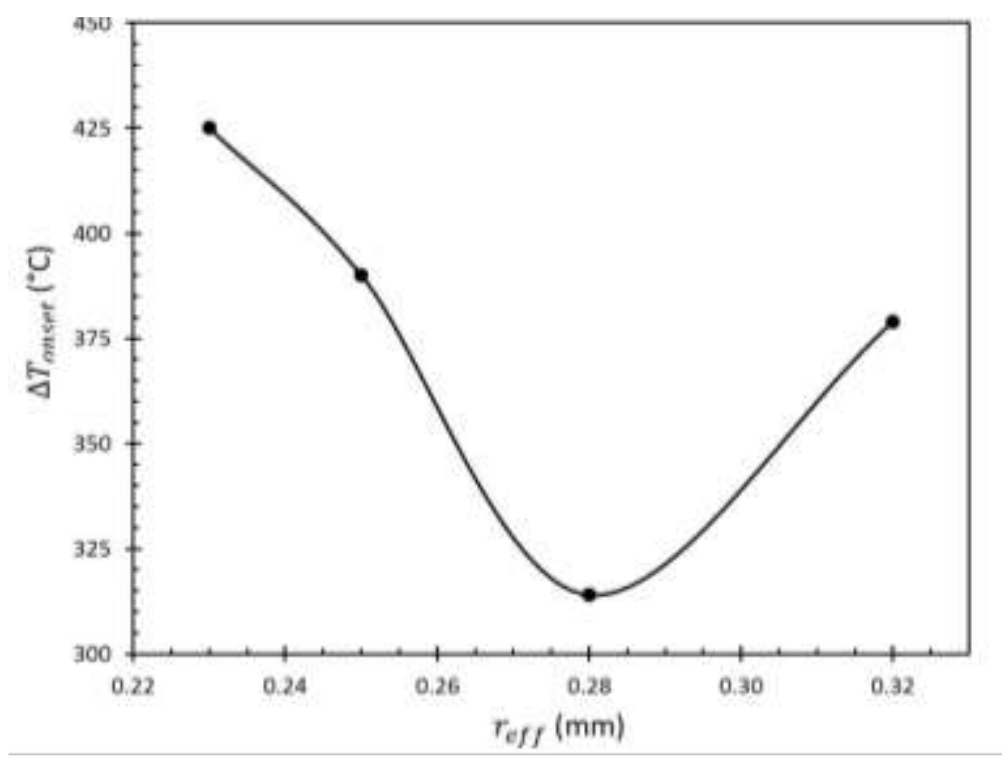

Figure 5. The onset temperature difference $\left(\Delta T_{\text {onset }}\right)$ for different effective hydraulic radiuses of stack $\left(r_{e f f}\right)$

The results of the effect of stack effective hydraulic radius on the onset temperature difference are shown in Figure 5. When the hydraulic radius enlarged from $0,23 \mathrm{~mm}$ to $0,28 \mathrm{~mm}$, the onset temperature difference decreased to the lowest point of $314^{\circ} \mathrm{C}$, then increased with the increasing stack hydraulic radius.

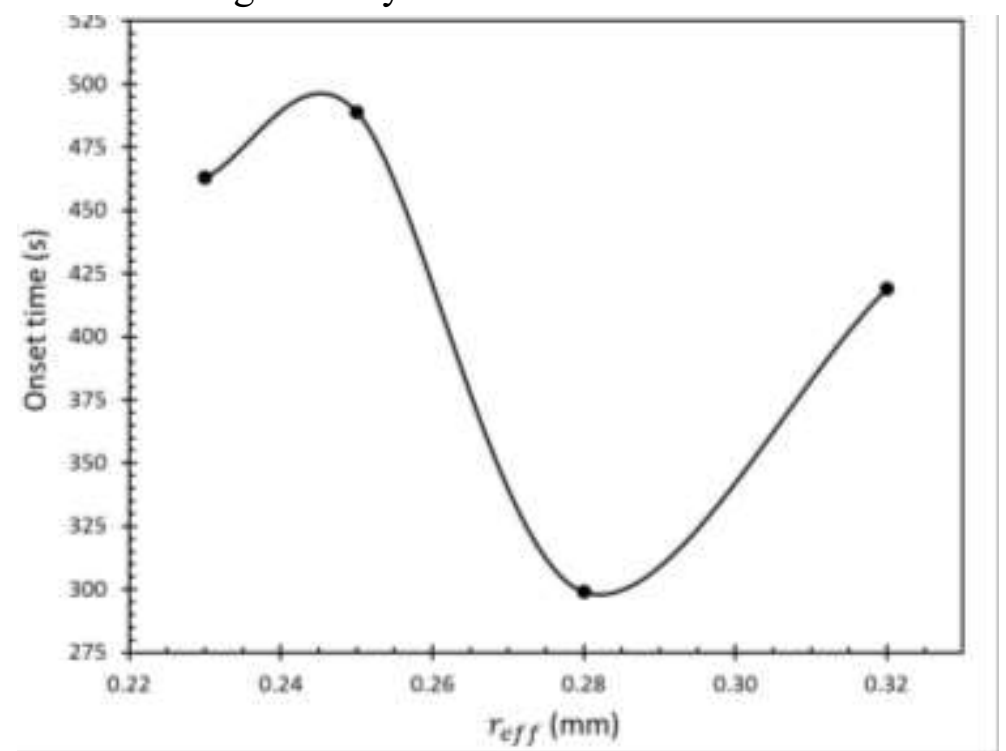

Figure 6. The onset time for various effective hydraulic radiuses $\left(r_{e f f}\right)$

The onset time is the time needed to generate sound waves starts from the electric heater turned on. At a glance, the curve pattern of onset time is not much different from the onset temperature difference pattern. The input power set to constant of 350 watts makes the heating rate at each hydraulic radius variation fixed, so that the onset time will be proportional to each of the onset temperature difference. The fastest time to reach onset condition is obtained when the hydraulic radius of stack is $0,28 \mathrm{~mm}$ for 299 
seconds. Low onset temperature difference and short onset time indicate that the heat required by prime mover to generate sound waves is few.

\subsection{Frequency of Sound Waves}

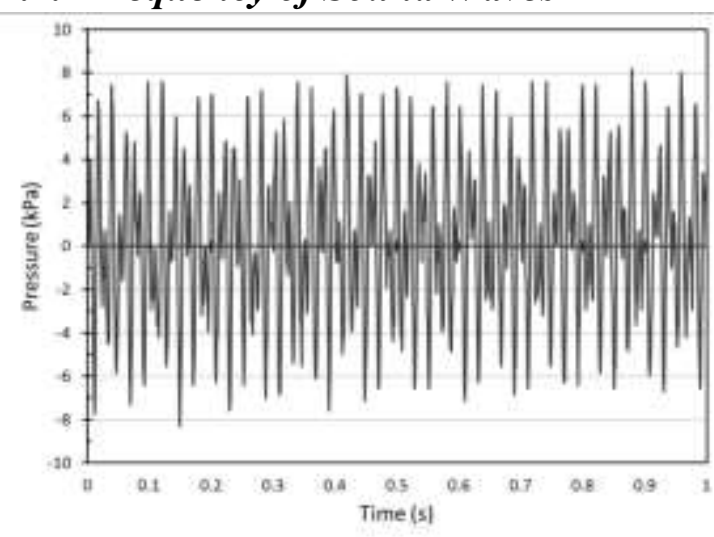

(a)

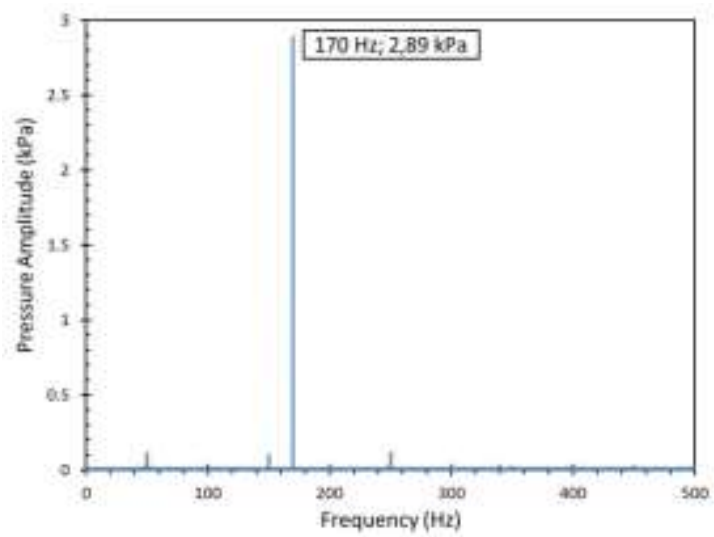

(b)

Figure 7. Sample data for (a) pressure oscillation of sound waves within 1 second, and (b) analysis result using Fast Fourier Transform method

Frequency and amplitude of the generated sound waves can be acquired from the pressure oscillation data at the pressure transducer processed using FFT method. Examples of pressure oscillation data and its result using FFT on prime mover with 0,28 $\mathrm{mm}$ stack hydraulic radius are shown in Fig. 7. Based on the result, it appears that the dominant frequency is $170 \mathrm{~Hz}$. In addition to these frequencies, there are other frequencies with relatively low amplitudes of $50 \mathrm{~Hz}, 150 \mathrm{~Hz}$, and $250 \mathrm{~Hz}$. These three frequencies are 'noises' coming from electricity due to bad grounding. The resonance frequency calculation using equation (9) in the second resonance order gives a value of $168 \mathrm{~Hz}$. This difference is caused by the presence of components inside the resonator pipe such as stack and heat exchanger.

Table 2. Frequency analysis results

\begin{tabular}{ccc}
\hline$r_{\text {eff }}(\mathbf{m m})$ & $\begin{array}{c}\text { Experiment } \\
\mathbf{( H z )}\end{array}$ & Calculation $\mathbf{( H z )}$ \\
\hline $\mathbf{0 , 3 2}$ & 170 & \\
$\mathbf{0 , 2 8}$ & 170 & 168 \\
$\mathbf{0 , 2 5}$ & 170 & \\
$\mathbf{0 , 2 3}$ & 172 & \\
\hline
\end{tabular}

The frequency analysis results on various mesh number of stack is summarized in Table 2. Based on the table, it appears that changes in hydraulic radius of stack does not affect much to the resulting frequency. In prime mover with effective hydraulic radius of $0,23 \mathrm{~mm}$, the sound frequency generated is slightly higher than the others, due to smaller and tighter pores on the stack, so that the effect of the stack presence becomes more dominant.

\subsection{Pressure Amplitude}


Pressure amplitude is half of the peaks of the pressure oscillation recorded using pressure transducer. The analyzed amplitude is the pressure amplitude at the moment of steady state. The sound waves pattern on the resonator with one open-end follows the $1 / 4$ wavelength pattern. According to the frequency analysis in the previous discussion, it is known that the resulting frequency is in the second resonance order, hence the pressure pattern along the resonator follows 3/4 wavelength pattern, as shown in Fig. 8. Based on 3/4 wavelength pattern, the two maximum pressure amplitudes (anti-node) are located in the closed-end of resonator and the other is in about $2 / 3$ times of the pipe length from the closed-end, while the two minimum pressure amplitudes (node) are located in the open-end of resonator and in about $1 / 3$ times of the pipe length from the closed-end.

Based on Fig. 8, if sorted according to the pressure amplitudes from high to low, the highest value obtained is $2,89 \mathrm{kPa}$ at hydraulic radius of $0,28 \mathrm{~mm}$, then followed by the hydraulic radius of $0,32 \mathrm{~mm}$ and $0,25 \mathrm{~mm}$, and the lowest is at hydraulic radius of 0,23 $\mathrm{mm}$. These results are in line with the results of onset temperature difference analysis. It shows that at the effective hydraulic radius of $0,28 \mathrm{~mm}$, interaction between working gas with the stack channel is optimal.

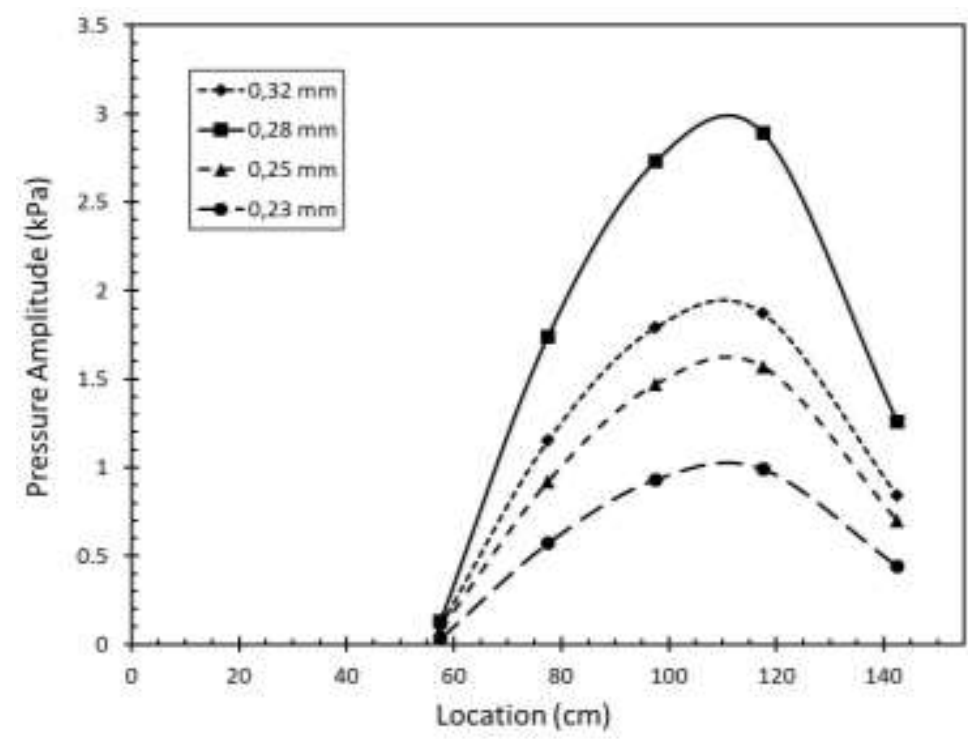

Figure 8. The pressure amplitudes pattern at various location along the resonator

\section{CONCLUSIONS}

Research on the effect of stack effective hydraulic radius on the performance of a thermoacoustic prime mover with one open-end resonator has been successfully performed. The lowest onset temperature difference, the fastest time to reach onset condition, and the highest amplitude of sound waves are obtained at the stack hydraulic radius of $0,28 \mathrm{~mm}$ using mesh number of \#14. Changes in the hydraulic radius value does not significantly affect the frequency of sound waves generated.

\section{REFERENCES}

Abduljalil, A. S., Yu, Z., \& Jaworski, A. J. (2011). Selection and Experimental Evaluation of Low-cost Porous Materials for Regenerator Applications in 
Thermoacoustic Engines. Materials and Design, 32, 217-228.

Agarwal, H., Unni, V. R., Akhil, K., Ravi, N., Iqbal, S. M., Sujith, R., et al. (2016). Compact Standing Wave Thermoacoustic Generator for Power Conversion Applications. Applied Acoustics, 110, 110-118.

Atchley, A. A. (1994). Analysis of The Initial Buildup of Oscillations in A Thermoacoustic Prime Mover. The Journal of the Acoustical Society of America, 95, 1661-1664.

Chen, R. L., \& Garret, S. L. (1998). Solar/Heat Driven Thermoacoustic Engine. The Journal of the Acoustical Society of America, 103, 813-814.

Gardner, D. L., \& Howard, C. Q. (2009). Waste-Heat-Driven Thermoacoustic Engine and Refrigerator. Proceedings of Acoustics.

Hao, X., Ju, Y., Behera, U., \& Kasthurirengan, S. (2011). Influence of Working Fluid on The Performance of A Standing-wave Thermoacoustic Prime Mover. Cryogenics, 51, 559-561.

Hariharan, N., Sivashanmugam, P., \& Kasthurirengan, S. (2015). Studies on Performance of Thermoacoustic Prime Mover. Experimental Heat Transfer, $28,267-281$.

Jin, T., Huang, J., Feng, Y., Yang, R., Tang, K., \& Radebaugh, R. (2015). Thermoacoustic Prime Movers and Refrigerators: Thermally Powered Engines Without Moving Components. Energy, 93, 828-853.

Murti, P. (2015). Studi Eksperimental Pengaruh Jejari Hidrolik dan Panjang Stack Terhadap Kinerja Prime Mover Termoakustik Gelombang Berdiri. Jurusan Teknik Mesin dan Industri. Yogyakarta: Universitas Gadjah Mada.

Normah, M., Irfan, A., Koh, K., Manet, A., \& Zaki, A. (2013). Investigation of A Portable Standing Wave Thermoacoustic Heat Engine. Procedia Engineering, $56,829-834$.

Rott, N. (1980). Thermoacoustics. Advances in Applied Mechanics, 20, 135-175.

Saechan, P. (2014). Application of Thermoacoustic Technologies for Meeting The Refrigeration Needs of Remote and Rural Communities in Developing Countries. Department of Engineering. Leicester: University of Leicester.

Setiawan, I., Murti, P., Utomo, A. B., \& Achmadin, W. N. (2015). Pembuatan dan Pengujian Prime Mover Termoakustik Tipe Gelombang Tegak. Proceeding Seminar Nasional Tahunan Teknik Mesin, XIV.

Ueda, Y. (2009). Experimental Evaluation of The Acoustic Properties of Stacked-screen Regenerators. The Journal of the Acoustical Society of America, 125, 780-786. 